\title{
Maneuvering Performance of a Ship with VecTwin Rudder System
}

\author{
by Masami Hamamoto*, Member Takashi Enomoto*, Member
}

\begin{abstract}
Summary
This paper is concerned with the maneuvering motion of a ship with VecTwin Rudder system. Firstly the forces on a couple of rudders and the interaction between both rudders steered with rudder angle are investigated analytically and experimentally. Secondly the free running model experiments are conducted for the turning, zig-zag and stopping maneuvers which are compared with the results simulated by mathematical model to describe the maneuvering motion. Finally an expression of force on Vec Twin Rudder is proposed for the MMG model.
\end{abstract}

\section{Introduction}

VecTwin Rudder system consists of a couple of rudders behind a propeller and a joystick steering device to control the rudder angle of each rudder separately. The rudders are movable to a very large angle compared with normal rudder, and have an unconventional section with wedge-shaped tail. By controlling the rudders to set the prescribed angle with out the change of propeller revolution, it is possible to give a ship several maneuvering mode which are called Ahead, Hovering, Astern, Ahead to starboard or port, Astern to starboard or port.

At the present time a number of ships which are equipped with VecTwin Rudder system has already operated as a shuttle tanker and carrier of dangerous cargo because of easy operation for maneuvering performance.

From a view point of operational safety of ships at sea, it will be an important subject to investigate and to make the properties of such maneuvering mode clear. The main problem here is to investigate the hydrodynamic property of VecTwin Rudder and to simulate several maneuvering mode by using the equations of maneuvering motion.

In order to obtain some solution of the problems, the following experimental and analytical approaches are carried out in this paper. Firstly, the wind tunnel tests were carried out for measuring the forces acting on a couple of rudders and the mutual interaction between both rudders. A theoretical approach based on Bollay's

* Osaka University

Received 10th Jan. 1997

Read at the Spring meeting 15th May 1997 flow model is made to analyze these experimental results. Secondly the free running test of a ship model with VecTwin Rudder system were conducted for evaluating the turning, course-keeping and stopping abilities. These maneuvering motions are analyzed in comparison with the simulated results which are obtained from a mathematical model to describe the maneuvering motion taking into account the properties of VecTwin Rudder system. Finally an expression of force on VecTwin Rudder is proposed for the MMG model $^{1)}$ as described in following. When a ship is traveling with constant forward speed is steered with rudder angles, the maneuvering motion of the ship is usually described in the coupled motions of swaying velocity $v$ and yawing angular velocity $r$ taking into account the variation of forward velocity $u$. As well known the equations of motion are:

$$
\begin{aligned}
& \left(m+m_{x}\right) \dot{u}-\left(m+m_{y}\right) v r \\
& \quad=T\left(1-t_{P}\right)-R(u)+X_{H}(v, r)-X_{R}\left(\delta_{1}, \delta_{2}\right) \\
& \left(m+m_{y}\right) \dot{v}+\left(m+m_{x}\right) u r=Y_{H}(v, r)-Y_{R}\left(\delta_{1}, \delta_{2}\right)
\end{aligned}
$$

$$
\left(I_{z}+J_{z}\right) \dot{r}=N_{H}(v, r)+N_{R}\left(\delta_{1}, \delta_{2}\right)
$$

where $\mathrm{m}$ and $I_{z}$ denote the mass and moment of inertia of a ship, $m_{x}, m_{y}$ and $J_{z}$ the longitudinal and lateral added masses and added moment of inertia respectively, $X_{H}, Y_{H}$ and $N_{H}$ the hydrodynamic forces and moment acting on a ship, $X_{R}, Y_{R}$ and $N_{R}$ the rudder forces and moment including the interaction between propeller and rudder, $\delta_{1}$ and $\delta_{2}$ the rudder angles of port and starboard sides, $T$ the thrust of propeller, $t_{P}$ the thrust deduction coefficient and $R(u)$ the ship resistance.

The expression and evaluation of the added masses and hydrodynamic forces in Eq. (1) are available from the empirical formula of Inoue and $\mathrm{Kijima}^{2)}$ or experimental data of the planar motion mechanism test and the circular motion test. Some new approach is how- 
ever required for the expression and evaluation of $X_{R}$, $Y_{R}$ and $N_{R}$ including the interaction with propeller and ship hull. Accordingly experimental and analytical approaches to the forces on VecTwin Rudder are mainly carried out in detail.

\section{Normal force on VecTwin Rudders}

In studying the properties of VecTwin Rudder system, Bollay's flow model ${ }^{3)}$ is applied for predicting the normal forces acting on the rudders and for investigating the mutual interaction of rudders. When the geometrical angles of incidence of both rudders are given as shown in Fig. 1, the rudders can be represented by vortex sheets $\gamma_{1}$ and $\gamma_{2}$ which have the same chord $\mathrm{c}$ and span $2 s$ as the rudder. According to Bollay's flow model, the vorticity is constant along the direction of span and varies with respect to the direction of chord. Thus the vorticities $\gamma_{1}(\xi)$ and $\gamma_{2}(x)$ are the function of the coordinate along the direction of chord where $\xi$ is the coordinate for the No. 1 rudder and $x$ for No. 2 rudder. The induced velocities normal to the center line of vortex sheet must be equal to the inflow velocity component normal to the vortex sheet and satisfies Kutta condition at the trailing edge. According to the flow model as shown in Fig. 1, the vorticities $\gamma_{1}(\xi)$ and $\gamma_{2}(x)$ of both rudders must satisfy the following integral equations :

$$
\begin{aligned}
& \frac{1}{2 \pi} \int_{0}^{c} \frac{\gamma_{1}(\eta)}{\xi-\eta} K_{11}(\xi, \eta) d \eta-\frac{1}{2 \pi} \int_{0}^{c} \gamma_{2}(x) K_{12}(\xi, x) d x \\
& \quad=U \sin \delta_{1} \\
& \frac{1}{2 \pi} \int_{0}^{c} \frac{\gamma_{2}(\eta)}{x-\eta} K_{22}(x, \eta) d \eta+\frac{1}{2 \pi} \int_{0}^{c} \gamma_{1}(\xi) K_{21}(\xi, x) d \xi \\
& \quad=U \sin \delta_{2}
\end{aligned}
$$

where $\eta$ is a integral variable, $\delta_{1}$ and $\delta_{2}$ rudder angles of No. 1 and No. 2 rudders, $K_{11}$ is the kernel function consisting of the bound and trailing vortices of No. 1 rudder, $K_{12}$ the kernel function consisting of induced velocity on No. 1 rudder due to bound and trailing vortices of No. 2 rudder, $K_{22}$ the kernel function for the effect of induced velocity of No. 2 rudder on No. 2 rudder, $K_{21}$ the kernel function for the mutual effect of induced velocity of No. 1 rudder on No. 2 rudder. The kernel function $K$ will be given as

$$
\begin{aligned}
K_{11}= & \frac{s}{\sqrt{(\xi-\eta)^{2}+s^{2}}-(\xi-\eta) \cos \delta_{1}} \\
K_{12}= & \frac{s}{\sqrt{f^{2}+s^{2}}}\left[\frac{h \sin \delta_{1}+(x-l) \cos \left(\delta_{1}-\delta_{2}\right)-(\xi-l)}{f^{2}}\right. \\
& \left.-\frac{\cos \delta_{1}}{\sqrt{f^{2}+s^{2}}+(x-l) \cos \delta_{2}-(\xi-l) \cos \delta_{1}}\right](5) \\
K_{22}= & \frac{s}{\sqrt{(x-\eta)^{2}+s^{2}}-(x-\eta) \cos \delta_{2}} \\
K_{21}= & \frac{s}{\sqrt{f^{2}+s^{2}}}\left[\frac{h \sin \delta_{2}+(x-l)-(\xi-l) \cos \left(\delta_{1}-\delta_{2}\right)}{f^{2}}\right. \\
& \left.+\frac{\cos \delta_{2}}{\sqrt{f^{2}+s^{2}}-(x-l) \cos \delta_{2}+(\xi-l) \cos \delta_{1}}\right]
\end{aligned}
$$

and $h$ is the gap of both rudders at the rudder stock, $l$ the distance measured from the rudder stock to the leading edge and $f$ the gap of between the point $\xi$ on the centerline of No. 1 rudder and the point $x$ on the centerline of No. 2 rudder which is

$$
\begin{aligned}
f^{2} & =h^{2}+(\xi-l)^{2}+(x-l)^{2}-2(\xi-l)(x-l) \cos \left(\delta_{1}-\delta_{2}\right) \\
& -2 h\left[(\xi-l) \sin \delta_{1}-(x-l) \sin \delta_{2}\right]
\end{aligned}
$$

It will be possible to obtain the numerical solution of integral equations ( 1 ) and (2) according to Sugai's method $^{4)}$ which is developed for a single wing. Applying this method to biplane system, the unknown functions $\gamma_{1}(\xi)$ and $\gamma_{2}(x)$ can be assumed as

$$
\begin{aligned}
& \gamma_{1}(\xi)=U \sin \delta_{1} \frac{1+\cos \theta}{\sin \theta}\left[\frac{A_{0}}{2}+\sum_{k=1}^{N} A_{k} \cos k \theta\right] \\
& \gamma_{2}(x)=U \sin \delta_{2} \frac{1+\cos \phi}{\sin \phi}\left[\frac{B_{0}}{2}+\sum_{k=1}^{N} B_{k} \cos k \phi\right]
\end{aligned}
$$
where

$$
\xi=\frac{c}{2}(1-\cos \theta) \quad 0 \leq \theta \leq \pi
$$

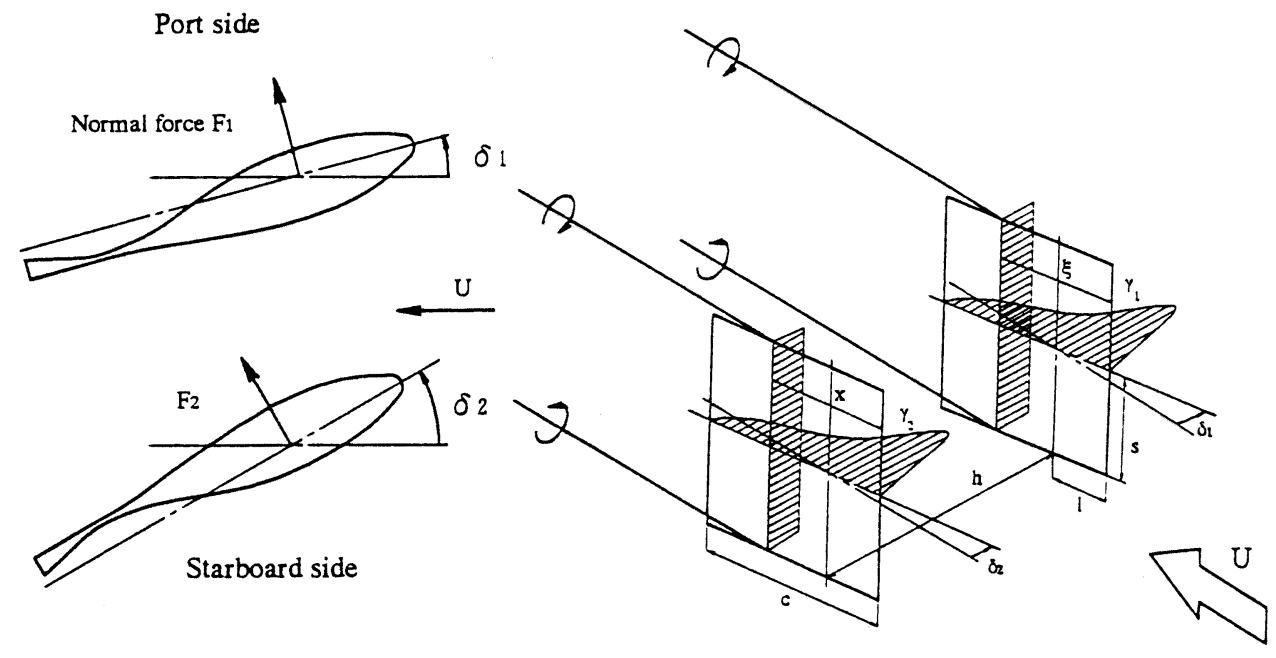

Fig. 1 Flow model for the VecTwin Rudder 


$$
x=\frac{c}{2}(1-\cos \phi) \quad 0 \leq \phi \leq \pi
$$

Substituting Eqs. (9), (10),(11) and (12) into Eqs. ( 2 ) and ( 3 ), Eqs. (2) and ( 3 ) can be derived into the simultaneous equations with respect to the unknown coefficients $A_{k}$ and $B_{k}$ which can be numerically determined from an infinite number of equations with an infinite number of unknowns. Thus the distribution of vorticities $\gamma_{1}(\xi)$ and $\gamma_{2}(x)$ can be evaluated Integrating $\gamma_{1}(\xi)$ and $\gamma_{2}(x)$ over the whole chord in accordance with Kutta-Joukowski theorem, the normal forces $F_{1}$ and $F_{2}$ acting on the rudders are obtained from the following equations :

$$
\begin{aligned}
& F_{1}=2 s \rho \int_{0}^{c} \gamma_{1}(\xi)\left[U \cos \delta_{1}+u_{11}+u_{12}\right] d \xi \\
& F_{2}=2 s \rho \int_{0}^{c} \gamma_{2}(x)\left[U \cos \delta_{2}+u_{22}-u_{21}\right] d x
\end{aligned}
$$

where $u_{11}$ is the tangential component to No. 1 rudder due to the trailing vorticices of No. 1 rudder, $u_{12}$ the tangential component to No. 1 rudder due to the induced velocities of No. 2 rudder, $u_{22}$ the tangential component to No. 2 rudder due to the trailing vorticices of No. 2 rudder, and $u_{21}$ the tangential component to No. 2 rudder due to the induced velocities of No. 1 rudder as follows :

$$
\begin{aligned}
& u_{11}=\frac{1}{2 \pi} \int_{0}^{c} \gamma_{1}(\eta) J_{11}(\xi, \eta) d \eta \\
& u_{12}=\frac{1}{2 \pi} \int_{0}^{c} \gamma_{2}(x) J_{12}(\xi, x) d x \\
& u_{22}=\frac{1}{2 \pi} \int_{0}^{c} \gamma_{2}(\eta) J_{22}(x, \eta) d \eta \\
& u_{21}=\frac{1}{2 \pi} \int_{0}^{c} \gamma_{1}(\xi) J_{22}(\xi, x) d \xi
\end{aligned}
$$

and the kernel functions $J_{11}, J_{12}, J_{22}$ and $J_{21}$ are

$$
\begin{aligned}
J_{11}= & \frac{s}{\sqrt{(\xi-\eta)^{2}+s^{2}}} \cdot \frac{\sin \delta_{1}}{\sqrt{(\xi-\eta)^{2}+s^{2}}-(\xi-\eta) \cos \delta_{1}} \\
J_{12}= & \frac{s}{\sqrt{f^{2}+s^{2}}}\left[\frac{h \cos \delta_{1}-(x-l) \sin \left(\delta_{1}-\delta_{2}\right)}{f^{2}}\right. \\
& \left.+\frac{\sin \delta_{1}}{\sqrt{f^{2}+s^{2}}+(x-l) \cos \delta_{2}-(\xi-l) \cos \delta_{1}}\right] \\
J_{22}= & \frac{s}{\sqrt{(x-\eta)^{2}+s^{2}}} \cdot \frac{\sin \delta_{2}}{\sqrt{(x-\eta)^{2}+s^{2}}-(x-\eta) \cos \delta_{2}} \\
J_{21}= & \frac{s}{\sqrt{f^{2}+s^{2}}}\left[\frac{h \cos \delta_{2}-(\xi-l) \sin \left(\delta_{1}-\delta_{2}\right)}{f^{2}}\right. \\
& \left.-\frac{\sin \delta_{2}}{\sqrt{f^{2}+s^{2}}-(x-l) \cos \delta_{2}+(\xi-l) \cos \delta_{1}}\right]
\end{aligned}
$$

In addition, when the angles of both rudders are the same as $\delta$, both kernel functions $K$ and $J$ became simpler in the expression as follows :

$$
\begin{aligned}
K_{11}= & \frac{s}{\sqrt{\xi-\eta)^{2}+s^{2}}-(\xi-\eta) \cos \delta} \\
K_{12}= & \frac{s}{\sqrt{f^{2}+s^{2}}}\left[\frac{h \sin \delta+(x-\xi)}{f^{2}}\right. \\
& \left.-\frac{\cos \delta}{\sqrt{f^{2}+s^{2}}+(x-\xi) \cos \delta}\right] \\
K_{22}= & \frac{s}{\sqrt{(x-\eta)^{2}+s^{2}}-(x-\eta) \cos \delta}
\end{aligned}
$$

$$
\begin{aligned}
K_{21}= & \frac{s}{\sqrt{f^{2}+s^{2}}} \\
& \times\left[\frac{h \sin \delta+(x-\xi)}{f^{2}}+\frac{\cos \delta}{\sqrt{f^{2}+s^{2}}-(x-\xi) \cos \delta}\right] \\
J_{11}= & \frac{s}{\sqrt{(\xi-\eta)^{2}+s^{2}}} \cdot \frac{\sin \delta}{\sqrt{(\xi-\eta)^{2}+s^{2}}-(\xi-\eta) \cos \delta} \\
J_{12}= & \frac{s}{\sqrt{f^{2}+s^{2}}}\left[\frac{h \cos \delta}{f^{2}}+\frac{\sin \delta}{\sqrt{f^{2}+s^{2}}+(x-\xi) \cos \delta}\right] \\
J_{22}= & \frac{s}{\sqrt{(x-\eta)^{2}+s^{2}}} \cdot \frac{\sin \delta}{\sqrt{(x-\eta)^{2}+s^{2}}-(x-\eta) \cos \delta} \\
J_{21}= & \frac{s}{\sqrt{f^{2}+s^{2}}}\left[\frac{h \cos \delta}{f^{2}}-\frac{\sin \delta}{\sqrt{f^{2}+s^{2}}-(x-\xi) \cos \delta}\right]
\end{aligned}
$$

and

$$
f^{2}=h^{2}+(x-\xi)^{2}+2(x-\xi) \sin \delta
$$

The normal force coefficients $C_{N 1}$ and $C_{N 2}$ of each rudder are given by

$$
C_{N 1}=\frac{F_{1}\left(\delta_{1}, \delta_{2}\right)}{1 / 2 \rho U^{2} A_{R}}, \quad C_{N 2}=\frac{F_{2}\left(\delta_{1}, \delta_{2}\right)}{1 / 2 \rho U^{2} A_{R}}
$$

where $A_{R}$ is the projected area of a rudder.

Since either of the coefficients is given as a function of $\delta_{1}$ and $\delta_{2}$, the influence of No. 2 rudder on the coefficient $C_{N 1}$ of No. 1 rudder can be evaluated from Eqs. (13) and (14). Fig. 2 shows the normal force coefficient $C_{N 2}$ versus rudder angles $\delta_{2}$ when No. 1 rudder was fixed with rudder angles $\delta_{2}=-25,0,25$ respectively. Fig. 3 indicates the normal and tangential force coefficients obtained from the wind tunnel tests in which both rudders are steered with the same angles in the range from-25 to 25 degrees. But only No. 2 rudder is steered with the angle over 25 degrees while No. 1 rudder is kept in the constant angle 25 degrees and for the negative rudder angle, then only No. 1 rudder is steered with the angle over-25 degrees while No. 2 rudder is kept in

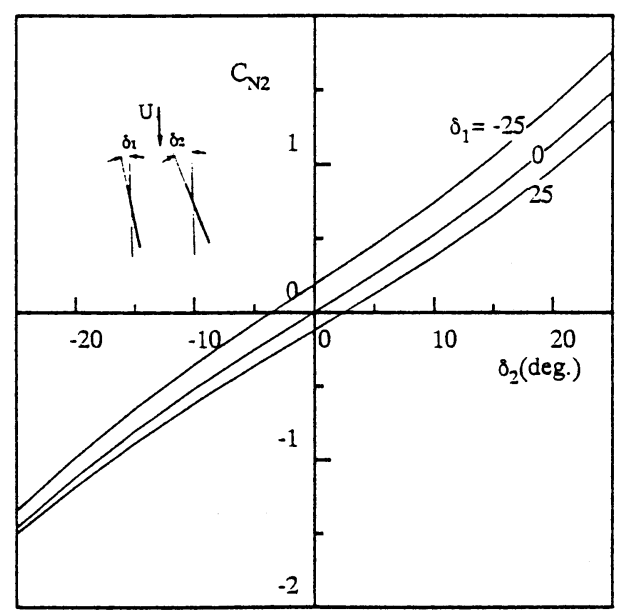

Fig. 2 Normal force coefficient of VecTwin Rudders 
constant angle-25 degrees. Besides the normal force coefficients obtained from the theoretical computation for a couple of rudders steered with the same angle are indicated in comparison with the experimental results. In Fig. 3 the experimental and theoretical results show a good agreement within the range of 20 degrees. But No. 1 rudder stalls out at a smaller angle than that of No. 2 rudder is opposite in the negative range of rudder angle because of the mutual interaction between a couple of rudders. Let us next consider a practical expression to describe the normal force coefficient for the equations of maneuvering motion. According to the theoretical analysis, it will be possible to obtain the slope of normal force coefficients taking into account the effect of mutual interaction between No. 1 and No. 2 rudders from the results shown in Figs. 2 and 3, which can be described as

$$
\begin{aligned}
& C_{N 1}=f_{11} \sin \delta_{1}-f_{12} \sin \delta_{2} \\
& C_{N 2}=f_{22} \sin \delta_{2}-f_{21} \sin \delta_{1}
\end{aligned}
$$
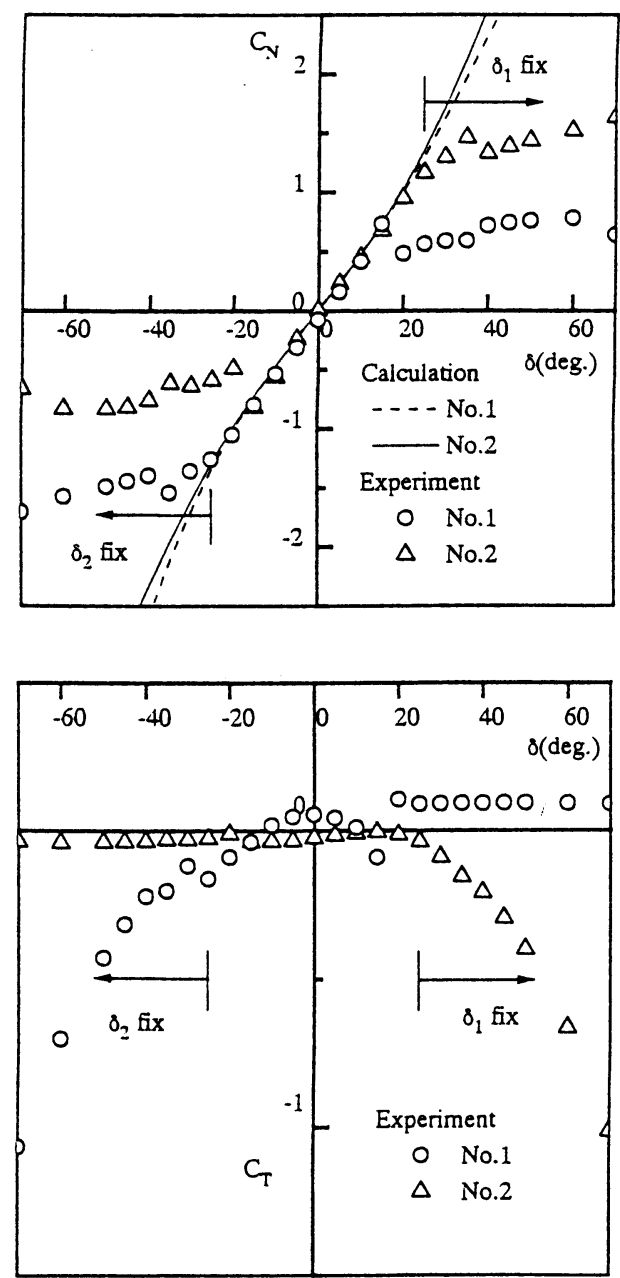

Fig. 3 Experimental results of normal and tangential coefficients where $f_{11}$ and $f_{22}$ are the slope of $C_{N 1}$ and $C_{N 2}, f_{12}$ and $f_{21}$ the interactions of No. 2 rudder to No. 1 rudder and No. 1 rudder to No. 2 rudder.

In Eqs. (33) and (34), the slope $f_{11}$ must equal to $f_{22}$ and the slope $f_{12}$ has to be equal to $f_{21}$ because they have to be the same slope as that of opposite rudder angle. Putting $f_{11}$ and $f_{22}$ to $f_{\alpha}$, and $f_{12}$ and $f_{21}$ to $\Delta f_{\alpha}, C_{N 1}$ and $C_{N 2}$ can be rewritten in the following forms:

$$
\begin{aligned}
& C_{N 1}=f_{\alpha} \sin \delta_{1}-\Delta f_{\alpha} \sin \delta_{2} \\
& C_{N 2}=f_{\alpha} \sin \delta_{2}-\Delta f_{\alpha} \sin \delta_{1}
\end{aligned}
$$

The theoretical results of $f_{\alpha}$ and $\Delta f_{\alpha}$ are compared with the experimental results from the wind tunnel test shown in Fig. 4. Although the normal force coefficients $C_{N 1}$ and $C_{N 2}$ vary with a non-linearity as a function of rudder angles, the average values of $f_{\alpha}$ and $\Delta f_{\alpha}$ are almost constant in the range of a small rudder angle where $f_{\alpha}$ is approximately ten times bigger than $\Delta f_{\alpha}$.

\section{Turning and Zig-Zag Maneuvers}

Free running model experiments were carried out for evaluating the turning and course-keeping abilities of a car carrier with VecTwin Rudder system as shown in Fig. 5 and Table 1. And also the time domain simulations were conducted by making use of the mathematical model to describe the hydrodynamic forces on the model and the rudder forces obtained from the previous section.

It is first required to evaluate the hydrodynamic forces $X_{H}, Y_{H}$ and moment $N_{H}$ as follows:

$$
\begin{aligned}
-R+ & X_{H}=-\frac{1}{2} \rho U^{2} L d\left[C_{T}\left(\frac{u}{U}\right)^{2}+X_{v r}\left(\frac{v}{U}\right)\left(\frac{L r}{U}\right)\right] \\
Y_{H}= & -\frac{1}{2} \rho U^{2} L d\left[Y_{v}\left(\frac{v}{U}\right)-Y_{r}\left(\frac{L r}{U}\right)+Y_{v v v}\left(\frac{v}{U}\right)^{3}\right. \\
& \left.+Y_{v r r}\left(\frac{v}{U}\right)\left(\frac{L r}{U}\right)^{2}-Y_{v v r}\left(\frac{v}{U}\right)^{2}\left(\frac{L r}{U}\right)\right] \\
N_{H}= & -\frac{1}{2} \rho U^{2} L^{2} d\left[N_{r}\left(\frac{L r}{U}\right)+N_{v}\left(\frac{v}{U}\right)\right. \\
& \left.-N_{v v v}\left(\frac{v}{U}\right)^{3}+N_{v r r}\left(\frac{v}{U}\right)\left(\frac{L r}{U}\right)^{2}-N_{v v r}\left(\frac{v}{U}\right)\left(\frac{L r}{U}\right)\right]
\end{aligned}
$$

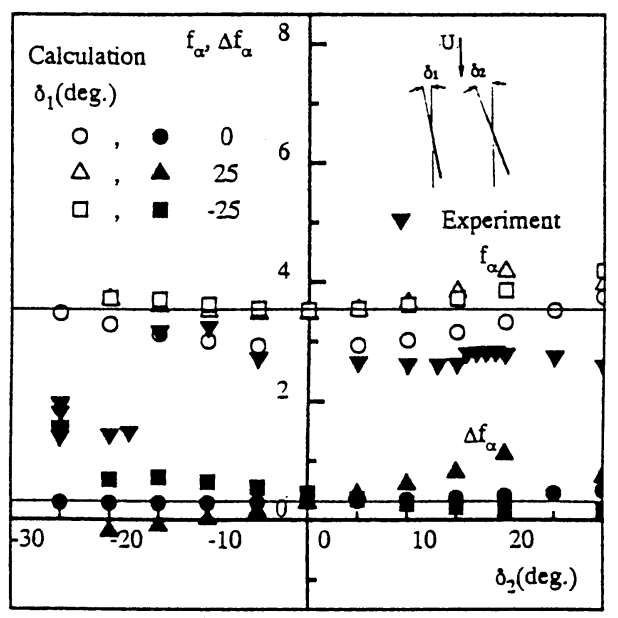

Fig. 4 Slope of the normal force coefficients 

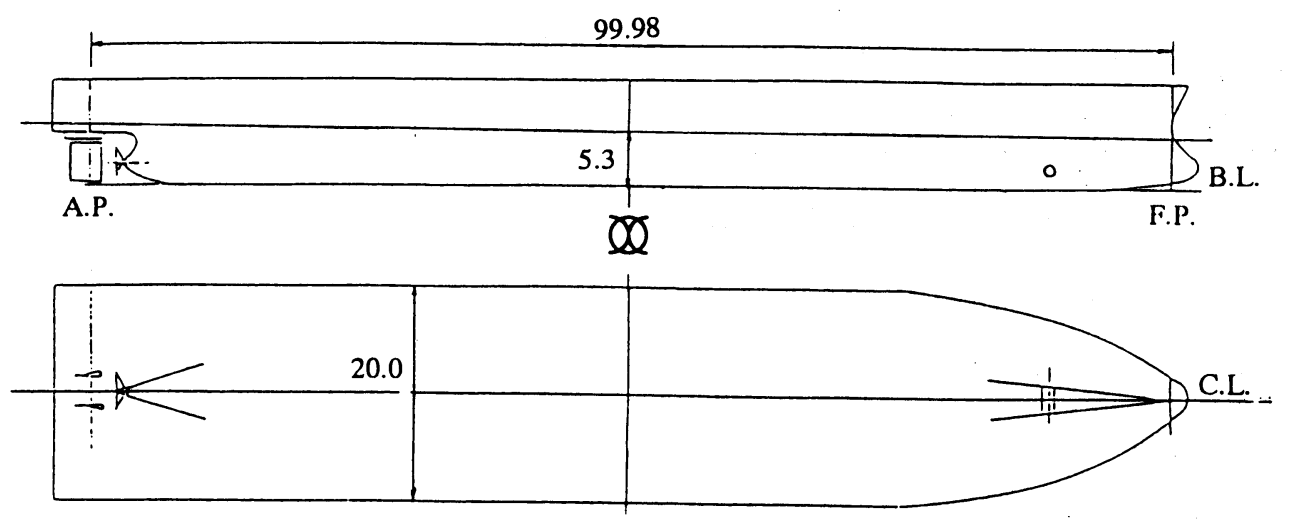

Fig. 5 General arrangement of Car Carrier with VecTwin Rudder system

Table 1 Principal dimension of Car Carrier

\begin{tabular}{|c|c|c|}
\hline & Ship & Model \\
\hline $\mathrm{L}_{\mathrm{PP}}(\mathrm{m})$ & 99.98 & 3.40 \\
\hline $\mathrm{B}(\mathrm{m})$ & 20.0 & 0.618 \\
\hline $\mathrm{d}_{\mathrm{F}}(\mathrm{m})$ & 4.8 & 0.1632 \\
\hline $\mathrm{d}_{\mathrm{M}}(\mathrm{m})$ & 5.3 & 0.1802 \\
\hline $\mathrm{d}_{\mathrm{A}}(\mathrm{m})$ & 5.8 & 0.1872 \\
\hline $\mathrm{TRIM}$ & $1 \%$ AFT & $1 \%$ AFT \\
\hline$\triangle($ tons,kg) & 5606 & 215.1 \\
\hline $\mathrm{S}\left(\mathrm{m}^{2}\right)$ & 2192 & 2.535 \\
\hline $\mathrm{L}_{\mathrm{WL}}(\mathrm{m})$ & 103.69 & 3.526 \\
\hline
\end{tabular}

where $C_{T}$ is the ship resistance coefficient, $Y_{v}, N_{v}, Y_{r}$ and $N_{r}$ are the linear hydrodynamic derivatives, and $X_{v r}, Y_{v v v}, Y_{v r r}, N_{v v v}, N_{v r r}$ and $N_{v v r}$ the non-linear hydrodynamic derivatives.

The ship resistance coefficient, the linear and nonlinear hydrodynamic derivatives are obtained from the towing test, oblique test, planar motion mechanism test and empirical formula of Inoue-Kijima as shown in Table 2. Next, according to MMG model, the rudder forces $X_{R}, Y_{R}$ and $N_{R}$ are represented as

$$
\begin{aligned}
& X_{R}=\left(1-t_{R}\right) \frac{1}{2} \rho A_{R} U_{R}^{2}\left[C_{N 1} \sin \delta_{1}+C_{N 2} \sin \delta_{2}\right] \\
& Y_{R}=\left(1+a_{H}\right) \frac{1}{2} \rho A_{R} U_{R}^{2}\left[C_{N 1} \cos \delta_{1}+C_{N 2} \cos \delta_{2}\right] \\
& N_{R}=\left(1+a_{R}\right) \frac{1}{2} \rho A_{R} U_{R}^{2} l_{R}\left[C_{N 1} \cos \delta_{1}+C_{N 2} \cos \delta_{2}\right]
\end{aligned}
$$

where $t_{R}, a_{H}$ and $a_{R}$ are the coefficients for mutual interaction between ship hull and rudders, $U_{R}$ the effective inflow velocity to the rudders, and $l_{R}$ the distance of rudder stock from the ship center of gravity. The effective inflow velocity $U_{R}$ taking into account the effect of swaying and yawing velocities is represented as
Table 2 Hydrodynamic coefficients

\begin{tabular}{|l|l|l|l|}
\hline & & & \\
\hline$m / \frac{1}{2} \rho L^{2} d$ & 0.207 & $m_{x} / \frac{1}{2} \rho L^{2} d$ & 0.0078 \\
$C_{T}$ & 0.0215 & $m_{y} / \frac{1}{2} \rho L^{2} d$ & 0.185 \\
$k_{z z}$ & 0.25 & $J_{z z} / \frac{1}{2} \rho L^{4} d$ & 0.0312 \\
$A_{R}\left(m^{2}\right)$ & 0.01048 & $Y_{v}$ & 0.311 \\
$1-w$ & 0.66 & $Y_{r}$ & 0.130 \\
$1-t_{P}$ & 0.76 & $Y_{\text {vvv }}$ & 0.0 \\
$1-t_{R}$ & 1.00 & $Y_{\text {vvr }}$ & 0.402 \\
$a_{H}$ & 0.25 & $Y_{\text {vrr }}$ & 0.749 \\
$a_{R}$ & 1.3 & $N_{v}$ & 0.150 \\
$l_{R}$ & 0.5 & $N_{r}$ & 0.046 \\
$\gamma$ & 0.5 & $N_{\text {vvv }}$ & 2.50 \\
$k$ & 0.5 & $N_{\text {vvr }}$ & 0.313 \\
& & $N_{\text {vrr }}$ & 0.133 \\
& & $X_{v r}$ & 0.102 \\
\hline
\end{tabular}

$$
\begin{aligned}
& U_{R}=\sqrt{u_{R}^{2}+v_{R}^{2}} \\
& u_{R}=u(1-w)\left[(1-k)+k \sqrt{1+\frac{8 K_{T}}{\pi J^{2}}}\right] \\
& v_{R}=\gamma v-l_{R} r
\end{aligned}
$$

where $u_{R}$ and $v_{R}$ are the longitudinal and lateral components of effective inflow velocity $U_{R}$ respectively, $w$ wake fraction, J advance constant, $K_{T}$ thrust coefficient, $\gamma$ flow straightening coefficient and $k$ acceleration rate of propeller.

The normal force coefficient $C_{N 1}$ and $C_{N 2}$ are decomposed as

$$
\begin{aligned}
& C_{N 1}=f_{\alpha} \sin \alpha_{R 1}-\Delta f_{\alpha} \sin \alpha_{R 2} \\
& C_{N 2}=f_{\alpha} \sin \alpha_{R 2}-\Delta f_{\alpha} \sin \alpha_{R 1}
\end{aligned}
$$

where $\alpha_{R 1}$ and $\alpha_{R 2}$ are the effective inflow rudder angle including the effect of swaying velocity and yawing angular velocity, as follows

$$
\alpha_{R 1}=\delta_{1}-\tan ^{-1} \frac{v_{R}}{u_{R}}
$$



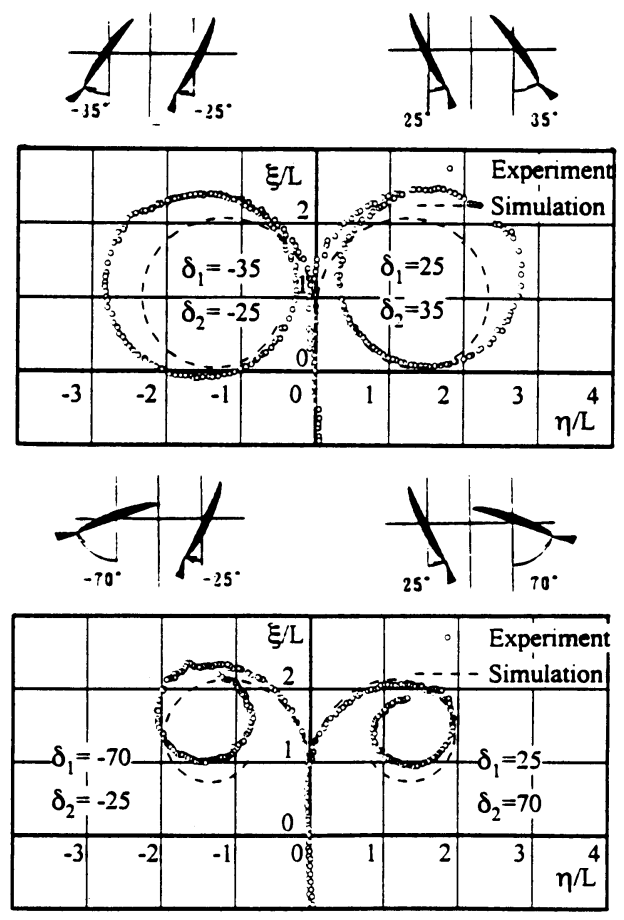

Fig. 6 Trajectories of turnning circle
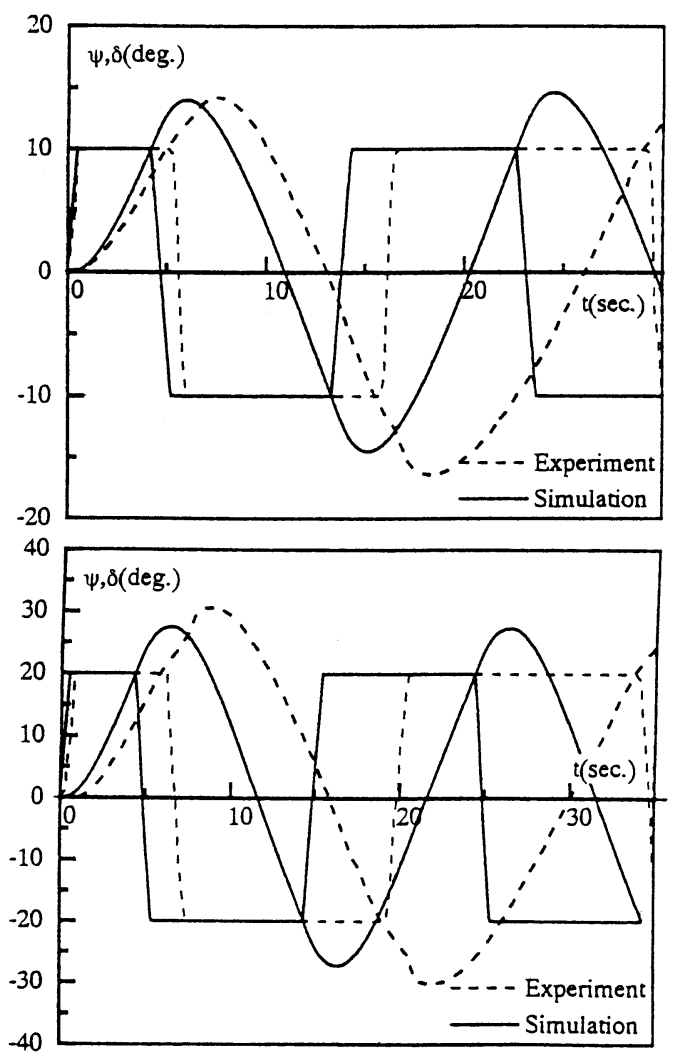

Fig. 7 Time histories of $10^{\circ}-10^{\circ}$ and $20^{\circ}-20^{\circ}$ zig-zag moneuver

$$
\alpha_{R 2}=\delta_{2}-\tan ^{-1} \frac{v_{R}}{u_{R}}
$$

Finally, substituting Eqs. (37) and (38) into Eq. (1), the equations of maneuvering motion can be arranged for the numerical simulation.

Figs. 6 shows the turning trajectories when ship was steered with the combination of rudder angle $\delta_{1}=-35$, 25 and $\delta_{2}=-25,35$, for the upper part, and $\delta_{1}=-70,25$ and $\delta_{2}=-25,70$ for the lower part of the Figures. Fig. 7 indicates the zig-zag maneuvers which are steered with the same angle as 10 and 20 degrees of model experiment in comparison with the simulations of them.

\section{Stopping Maneuvers}

The stopping maneuvers of VecTwin Rudder system are made by the rudder steered with the clam shell rudder angle as shown in Fig. 8. In this maneuvering mode, since the ship resistance remarkable increase in amount due to the drag of rudders behind a propeller, the ship speed decreases in a relatively short time. Two kind of experiments were carried out for evaluating the stopping ability. The first one is the wind tunnel tests to measure the force on rudders steered with the clam shell rudder angle. The second one is the free running model experiment to investigate the speed drop during stopping maneuver.

The wind tunnel tests were carried out on the basis of an analysis with respect to a single equation of forward speed $U$ in Eq. ( 1 ) which is

$$
\left(m+m_{x}\right) \frac{d U}{d t}=T\left(1-t_{p}\right)-R(U)-X_{R}\left(\delta_{c}\right)
$$

and

$$
\begin{aligned}
& T=\rho D^{2}(n P)^{2} K_{T} \\
& R=\frac{1}{2} \rho L d U^{2} C_{T} \\
& X_{R}=\left(1-t_{R}\right) \rho U_{R}^{2} A_{R} C_{D}\left(\delta_{C}\right)
\end{aligned}
$$

where $C_{D}\left(\delta_{C}\right)$ the drag coefficient of the rudders steered with clam shell rudder angle $\delta_{c}, n$ the propeller revolution and $P$ the propeller pitch.

When the ship is steered with the clam shell rudder

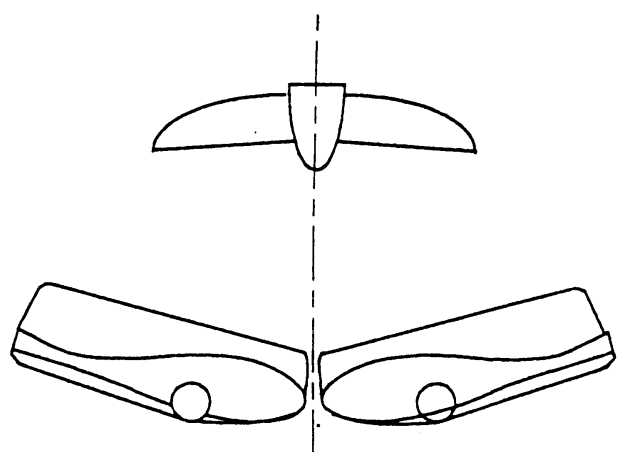

Fig. 8 Arrangement of VecTwin Rudder system at clam shell rudder angles 
angle, the ship speed decreases gradually to stop and goes astern after stopping because the flow accelerated by propeller is intercepted by the rudders. In this condition, the thrust reduction $t_{P}$ may remarkably change and be assumed to be larger than 1 . On the basis of this assumption, Eq. (45) can be rewritten as

$$
\frac{d U}{d t}=\frac{T}{m+m_{x}}\left[\left(t_{P}-1\right)+C\left(\frac{U}{n P}\right)^{2}\right]
$$

where

$$
C=\frac{L d C_{T}}{2 D^{2} K_{T}}+\left(1-t_{R}\right) \frac{A_{R} C_{D}}{D^{2} K_{T}}\left(\frac{U_{R}}{U}\right)^{2}
$$

From Eq. (47) the velocity $U$ during the stopping maneuver, the stopping time $t_{s}$ and the stopping distance $S$ are given by

$$
\begin{aligned}
\frac{U}{U_{0}}= & \frac{n P}{U_{0}} \sqrt{\frac{t_{P}-1}{C}} \tan \left[\tan ^{-1} \frac{U_{0}}{n P} \sqrt{\frac{C}{t_{P}-1}}\right. \\
& \left.-\frac{T \sqrt{C\left(t_{P}-1\right)}}{\left(m+m_{x}\right) n P} t\right]
\end{aligned}
$$
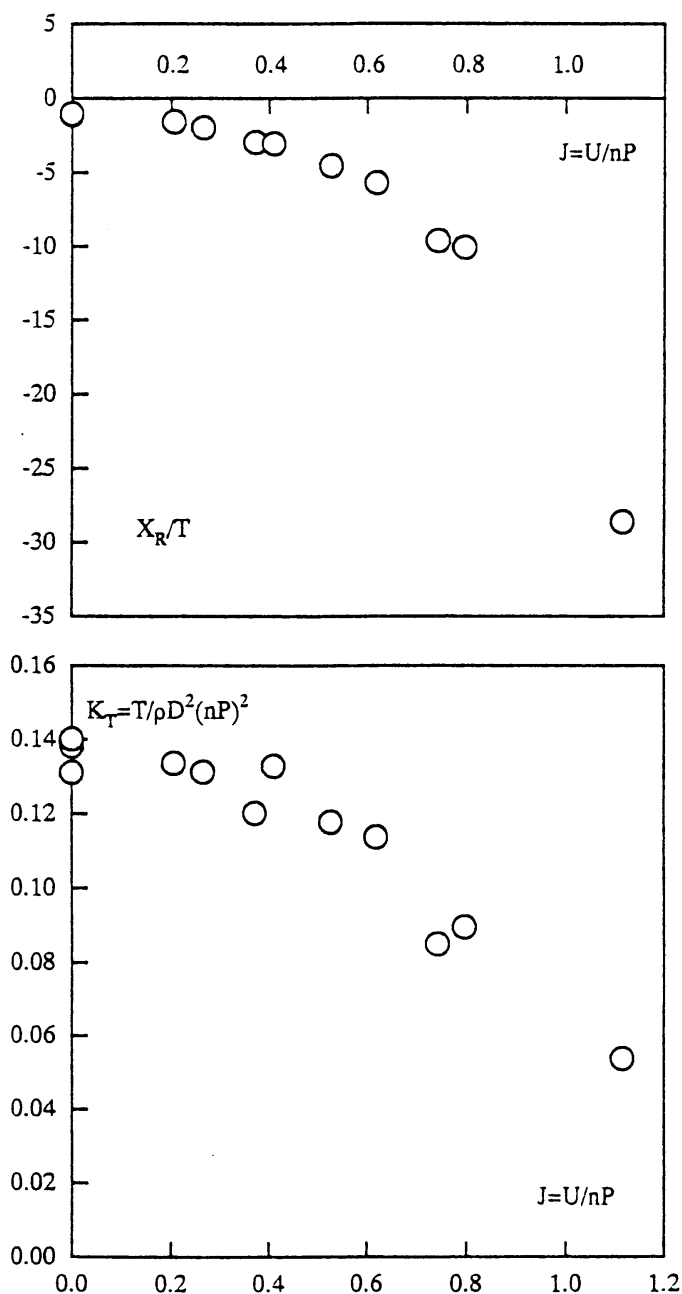

Fig. 9 Experimental results of $X_{R} / T$ and $K_{T}$ for advance constant

$$
\begin{aligned}
& t_{s}=\frac{\left(m+m_{x}\right) n P}{T \sqrt{C\left(t_{P}-1\right)}} \tan ^{-1} \frac{U_{0}}{n P} \sqrt{\frac{C}{t_{P}-1}} \\
& S=\frac{\left(m+m_{x}\right)(n P)^{2}}{2 C T} \log \left[1+\frac{C}{t_{P}-1}\left(\frac{U_{0}}{n P}\right)^{2}\right]
\end{aligned}
$$

Here $X_{R}$ and $\left(t_{P}-1\right)$ in Eq. (45) are unkown terms to be obtained from the experiments. $X_{R}$ can be evaluated by the wind tunnel tests measuring the force on the rudders steered with clam shell rudder angle behind the propellar as shown in Fig. 8. Fig. 9 indicates $X_{R} / T$ and $K_{T}$ for $U / n P$. Fig. 10 indicates the time history of speed drop $U / U_{0}$ maneuver and stopping distance $\xi / L$ during the stopping maneuver in comparison with the simulation from Eq. (49).
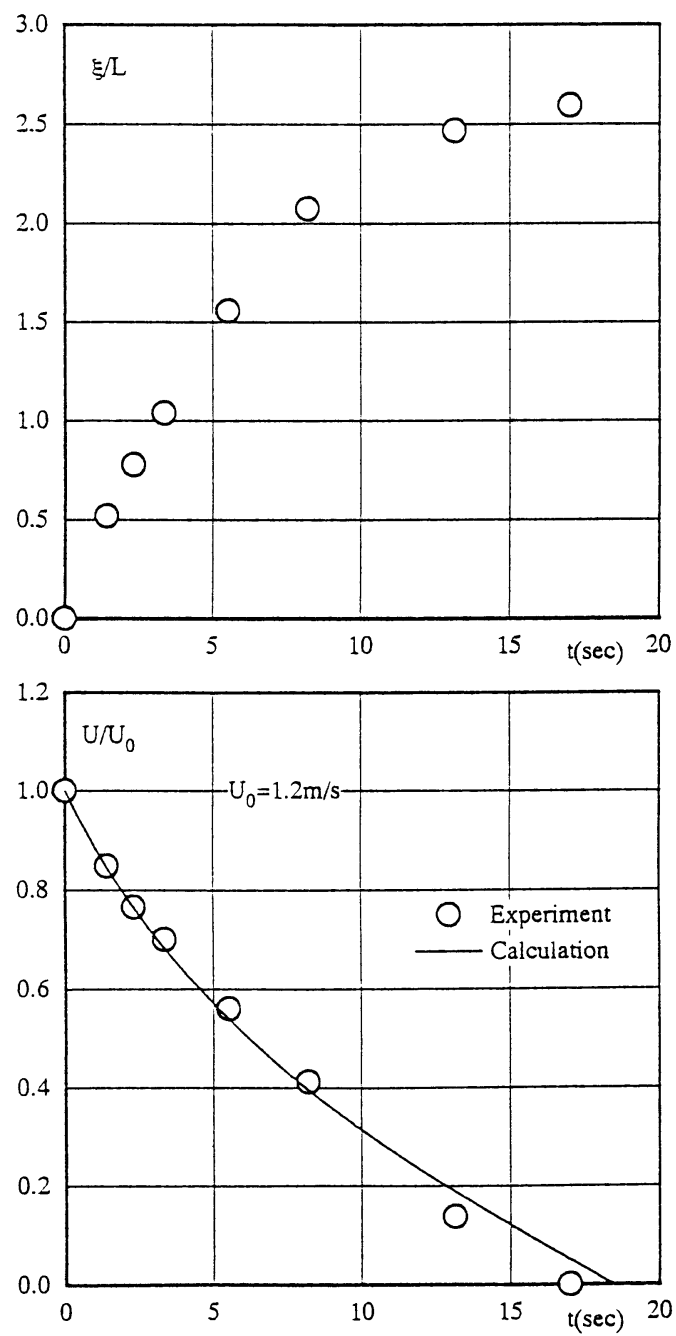

Fig. 10 Time histories of speed drop and stopping distance during stopping maneuver 


\section{Conclusions}

The experimental and analytical investigations are conducted for the maneuvering motion of a ship with VecTwin Rudder system. The main conclusions are summarized as follows:

(1) A practical formula to describe the normal force on a couple of steered rudders is presented on the basis of theoretical and experimental analysis taking into account the interaction between both rudders.

(2) The properties of turning, zig-zag and stopping maneuvers are evaluated by the free running model experiments and numerical simulations.

(3) An analytical formula to predict the speed drop during stopping maneuver, stopping time and stopping distance are proposed for a ship which is steered with the clam shell rudder angle.
This research has been supported by Japan Hammworthy\&Co., Ltd. and the scientific grant No. 07555312 (Prof. Kijima) from the Ministry of Education, Japan.

\section{References}

1) Proceedings of the $3 \mathrm{rd}$ Symposium on Ship Manoeuvrability, Society of Naval Architect of Japan, (1981)

2) Inoue, S., Hirano, M., Kijima, K. : Hydrodynamic derivatives on Ship monoeuvring, I. S. P., Vol. 28, No. 321, (1981)

3) Bollay, W.: A Non-linear Wing Theory and its application to Rectangular Wing of Small Aspect Ratio, Z. angew. Math. Mech, Bd. 19 Nr. 1, (1939), pp. 21-35.

4) Sugai, K.: A linear Approximation for the Lifting Surface with Low Aspect Ratio, Journal of the Society of Naval Architect of Japan, Vol. 117, (1965), pp. 37-49. 